\title{
Evaporation of Black Hole Under the Effect of Quantum Gravity
}

\author{
Riasat Ali, ${ }^{1, *}$ Rimsha Babar, ${ }^{2,+}$ Muhammad Asgher, ${ }^{3, \ddagger}$ and Syed Asif Ali Shah ${ }^{4, \S}$ \\ ${ }^{1}$ Department of Mathematics, GC University Faisalabad Layyah Campus, Layyah-31200, Pakistan \\ ${ }^{2}$ Division of Science and Technology, University of Education, Vehari, Lahore-54590, Pakistan \\ ${ }^{3}$ Department of Mathematics, The Islamia University of Bahawalpur, Bahawalpur-63100, Pakistan \\ ${ }^{4}$ Department of Mathematics and Statistics, The University of Lahore 1-Km Raiwind Road, Sultan Town Lahore 54000, Pakistan
}

(Dated: October 22, 2021)

\begin{abstract}
This paper provides an extension for Hawking temperature of Reissner-Nordström-de Sitter (RNDS) black hole (BH) with global monopole as well as 5D charged black hole. We consider the black holes metric and investigate the effects of quantum gravity $(\alpha)$ on Hawking radiation. We investigate the charged boson particles tunneling through the horizon of black holes by using the Hamilton-Jacobi ansatz phenomenon. In our investigation, we study the quantum radiation to analyze the Lagrangian wave equation with generalized uncertainty principle and calculate the modified Hawking temperatures for black holes. Furthermore, we analyze the charge and correction parameter effects on the modified Hawking temperature and examine the stable and unstable condition of RN-DS BH with global monopole as well as 5D charged black hole.
\end{abstract}

Keywords: Lagrangian Gravity Equation; Charged Black Hole; Boson Particles Tunneling.

\section{INTRODUCTION}

Hawking examined that black hole $(\mathrm{BH})$ emit thermal radiations because of the quantum vacuum volatility effects around the event horizon [1]. Hawking radiation can be defined by quantum tunneling method near the horizon of BHs. The Hawking radiation was determined as the black body radiation. This scenario indicates that the $\mathrm{BH}$ evaporates completely [2]. The correctness of the Hawking formula can be seen in different deductions dependent on the relativity quantum mechanics as well as quantum theory in curved space-time [3]. An effective model for studying the Hawking radiation is the semi-classical tunneling approach [4]-[14]. By taking into account the different spacetime in the background for this method, one can compute the corrected Hawking temperature. The generalized $\mathrm{BH}$ radiate all types of particles such as fermion, boson and scalar particles. The standard Hawking temperature as well as corrected temperature for such types of particles in the background of various space-time have been analyzed [15]-[18]. The corrected temperature rate is greater than the standard temperature, which means that the different background geometries accelerates the evaporation process.

However, the presence of a minimum observable length is predicted by different theories of quantum gravity, i.e., loop and string theories [19-21]. The generalized uncertainty Principle (GUP) is a clear way of understanding this minimum observable length. In order to determine the GUP, the basic commutation relations ought to be generalized. There are two different approaches to generalize these relations, which lead to various interpretations of GUP . Kempf et al. have put forward one way and derived the basic length [22]. Although, Das and his collaborators re-generalized the commutation relations on the basis of the theory of doubly special relativity as well as determined an equation of GUP [23]. The maximum observable momentum and the minimum observable length can be represented by the following expression [24]

$$
\left[\tilde{x}_{j}, \tilde{p}_{k}\right]=\imath \hbar\left[1+\tilde{p}^{2} \alpha\right] \delta_{j k}
$$

However, the GUP relation can be defined as

$$
\Delta \tilde{x} \Delta \tilde{p} \geq \frac{\hbar}{2}\left[1+(\Delta \tilde{p})^{2} \alpha\right]
$$

\footnotetext{
*Electronic address: riasatyasin@gmail.com

${ }^{\dagger}$ Electronic address: rimsha.babar10@gmail.com

‡Electronic address: m.asgher145@gmail.com

§Electronic address: asifalishah695@gmail.com
} 
here $\alpha=\frac{\alpha_{o}}{M_{\tilde{p}}^{2}}$. The $\alpha_{0}<10^{34}$ denotes the dimensionless parameter and $M_{\tilde{p}}$ represents the Plank's mass. Moreover, $\tilde{x}_{j}, \tilde{p}_{k}$ can be represented as $\tilde{x}_{j}=\tilde{x}_{0 j}$ and $\tilde{p}_{k}=\tilde{p}_{0 k}\left(1+\tilde{p}^{2} \alpha\right)$ whereas $\tilde{x}_{0 j}$ as well as $\tilde{p}_{0 k}$ agrees the relation $\left[\tilde{x}_{0 j}, \tilde{p}_{0 k}\right]=$ $i \hbar \delta_{j k}$. The generalization of the basic commutation relations is not specific. Several generalizations of commutation relations are alluded to [25]-[27]. In order to obtain some knowledge about the quantum properties of gravity, these modifications are applied extensively. Black holes are useful tools for investigating the quantum gravity effects. Some interesting consequences and findings were obtained by the establishment of quantum gravity effects into $\mathrm{BH}$ physics by incorporating GUP [17]. Furthermore, the remnant mass and corrections to the Hawking temperature and entropy were also analyzed. Övgün and Jusufi [28] have analyzed the GUP effects to massive spin-1 and spin-0 particles in the background of warped DGP gravity $\mathrm{BH}$.

The surface gravity and Hawking radiation by the first law of thermodynamics through the quantum horizon has been described [29]. The results between Hawking radiation and surface gravity of a BH have been compared. The tunneling of massive charged particles with the emission via first law of $\mathrm{BH}$ thermodynamics has been analyzed [30]. The author calculated the tunneling probability through the framework of non-commutative quasi coordinates of Reissner-Nordström (RN) BH.

Sharif and Abbas [31] analyzed the phantom energy accretion at $\mu<0$ and formulated equation of motion for steady state spherically symmetric spacetime. Furthermore, they discuss about the accretion and critical accretion of $\mathrm{BH}$. They concluded that the mass of $\mathrm{BH}$ decreases due to phantom accretion. Ling [32] extended the fermion tunneling method to 5D black lenses. As a consequence of their analysis they argued that with the help of tunneling phenomenon one can find correct values of Hawking temperature for static and rotating $\mathrm{BH}$. Liu et al. [33] investigated the quantum corrections, spacetime and energy conservation of charged particles tunneling in a modified Reissner BH. He found that the entropy does not depend on the dispersion relations of matter fields. In [34], the authors derive the geodesic equations of massive particles in a decent manner. They also derive Hawking radiation via tunneling from charged black hole $(\mathrm{CBH})$. Generalized uncertainty principle effect on Hawking radiation via $\mathrm{BH}$ geometry was studided by Gecim and Sucu [35]. From this analysis they saw that the Hawking temperature increases when angular momentum increases. Shababi and Addazi [36] have analyzed the cosmological vacuum energy, potential and quantum results on new type of higher D-dimensional nonperturbative GUP models. They have [37] also discussed the nonperturbative and quantum mechanics from GUP for D-dimension and non-commutative space. The nonlinear quantum algebra extension and functional momenta operator has been extended to the GUP and non-commutative algebra spacetime [38]. Khosropour and his colleagues [39] have also investigated the nonlinear Schrodinger and non-linear Klein-Gordon equations in the framework of GUP. Many important contributions have been made on extended gravity and teleparallel gravity for different BHs [40]-[43]. The total momentum and energy of an isolated system has been extracted in integral surface over a closed large surface enclosing the system as well as the form of an asymptotics of parallel vector fields at distance considerable from the source and the weak gravitational field have been studied and the most general linear gravitational Lagrangian field equation has been followed in vacuum [44].

The main objective of this paper is to provide the extension of Hawking temperature for spin-1 particles near the event horizon from RN-de Sitter (DS) BH with global monopole as well as 5D CBH by taking into account the quantum gravity effects. Firstly, we modify the Proca equation under the influence of quantum gravity. To meet our goal, we follow the GUP framework. In order to study the equation of motion for spin-1 particles, we use the Hamilton-Jacobi process. After this, we calculate the modified tunneling probability and corrected Hawking temperatures. Moreover, we check the stability condition of BHs via graphical interpretation of temperature with event horizon.

We have organized our paper as follows: In Sec. II, we have investigated the surface gravity and quantum gravity effect on modified tunneling probability and corrected Hawking temperature of RN-DS BH with global monopole. Sec. III, we have investigated the graphical behavior of modified temperature w.r.t horizon to study the quantum gravity and charge effects on BH. Sec. IV discuss the corrected temperature for 5D CBH. In Sec. V, we discuss the effects of charge and gravity on modified temperature with graphs. In the last Sec. VI, we have summarized our results.

\section{RESSNER-NORDSTRÖM-DE-SITTER BLACK HOLE WITH GLOBAL MONOPOLE}

The most valuable predictions of general relativity (GR) are BHs. The first nontrivial BH solution of the Einstein field equations is called the Schwarzschild $\mathrm{BH}$ and its extension with charge version is known as the RN BH. The exploration of rotating case is familiar as Kerr and Kerr-Newman BH. After, these BHs have been described by incorporating various sources such as acceleration, magnetic charge and cosmological constant to the usual $\mathrm{BH}$ mass. The RN-DS BH with global monopole is the most fascinating extension of the solution of Einstein field equations. 
The spacetime with a global monopole parameter of the RN-DS BH can be given as [45]

$$
d s^{2}=-A(r) d t^{2}+\frac{1}{A(r)} d r^{2}+B(r) d \theta^{2}+C(r) d \phi^{2},
$$

where

$$
\begin{aligned}
& A(r)=1+\frac{q^{2}}{r^{2}}-\frac{2 m}{r}-\left(\frac{\Lambda}{3}\right) r^{2}, \\
& B(r)=\left(1-8 \pi \eta^{2}\right) r^{2}, \\
& C(r)=\left(1-8 \pi \eta^{2}\right) r^{2} \sin ^{2} \theta,
\end{aligned}
$$

here $\eta, \Lambda, q$ and $m$ represents the global monopole, cosmological constant, $\mathrm{BH}$ charge and mass, respectively. The $\mathrm{BH}$ event horizon $r_{+}$can be determined from setting $A\left(r_{+}\right)=0$. Furthermore, in the presence of global monopole parameter, the total mass of Arnowitt-Deser-Misner and BH charge can be defined as [46]

$$
M=m\left(1-8 \pi \eta^{2}\right), Q=q\left(1-8 \pi \eta^{2}\right) .
$$

In generally, the gravity parameter is related with the property of $\mathrm{BH}$ stability. The physical importance of the GUP parameter in the Lagrangian equation has been analyzed [8]. As a Lagrangian field equation, the GUP parameter is the generalization of the field equation without singularity. To investigate the radiation phenomenon for massive bosons, we use the modified GUP Lagrangian equation with vector field $\chi^{\mu}$ for bosons particles can be defined as $[47,48]$

$$
£^{G U P}=\frac{1}{2}\left(D_{\mu} \chi_{v}-D_{\nu} \chi_{\mu}\right)\left(D^{\mu} \chi^{\nu}-D^{v} \chi^{\mu}\right)-\frac{1}{h} e F^{v \mu} \chi_{\mu} \chi_{v}-\frac{m^{2}}{h^{2}} \chi_{\mu} \chi^{v}
$$

The modified field equation can be expressed [8] as follows

$\partial_{\mu}\left(\sqrt{-g} \chi^{v \mu}\right)+\sqrt{-g} \frac{m^{2}}{\hbar^{2}} \chi^{v}+\sqrt{-g} \frac{i}{\hbar} e A_{\mu} \chi^{\nu \mu}+\sqrt{-g} \frac{i}{\hbar} \chi_{\mu} e F^{\nu \mu}+\hbar^{2} \partial_{0} \partial_{0} \partial_{0}\left(\sqrt{-g} g^{00} \chi^{0 v}\right)-\alpha \hbar^{2} \partial_{i} \partial_{i} \partial_{i}\left(\sqrt{-g} g^{i i} \chi^{i v}\right)=0$,

here $g$ shows the determinant of coefficient matrix, $\chi^{\nu \mu}$ represents the anti-symmetric tensor and $m$ gives the particle mass. The anti-symmetric tensor $\chi_{\nu \mu}$ can be denoted as

$$
\begin{aligned}
& \chi_{v \mu}=\left(1-\hbar^{2} \alpha \partial_{\nu}{ }^{2}\right) \partial_{\nu} \chi_{\mu}-\left(1-\hbar^{2} \alpha \partial_{\mu}{ }^{2}\right) \partial_{\mu} \chi_{\nu}+\left(1-\hbar^{2} \alpha \partial_{\nu}{ }^{2}\right) \frac{i}{\hbar} e A_{\nu} \chi_{\mu}-\left(1-\hbar^{2} \alpha \partial_{\mu}{ }^{2}\right) \frac{i}{\hbar} e A_{\mu} \chi_{v}, \\
& \text { and } \\
& F_{v \mu}=\nabla_{v} A_{\mu}-\nabla_{\mu} A_{\nu}, \quad \nabla_{o}=\left(1+\hbar^{2} \alpha g^{00} \nabla_{o}^{2}\right) \nabla_{o}, \quad \nabla_{i}=\left(1-\hbar^{2} \alpha g^{i i} \nabla_{i}^{2}\right) \nabla_{i},
\end{aligned}
$$

here $\alpha$ is quantum gravity (GUP parameter), $A_{\mu}, e$ and $\nabla_{\mu}$ are the $\mathrm{BH}$ potential, charge of emitted particle and covariant derivative, respectively. The elements of $\chi^{\mu}$ and $\chi^{\mu v}$ is defined as

$$
\begin{aligned}
& \chi^{0}=-\frac{1}{A} \chi_{0}, \quad \chi^{1}=A \chi_{1}, \quad \chi^{2}=\frac{1}{B} \chi_{2}, \quad \chi^{3}=\frac{1}{C} \chi_{3}, \quad \chi^{01}=-\chi_{01}, \\
& \chi^{02}=-\frac{1}{A B} \chi_{02}, \quad \chi^{03}=-\frac{1}{A C} \chi_{03}, \quad \chi^{12}=\frac{A}{B} \chi_{12}, \quad \chi^{13}=\frac{A}{C} \chi_{13}, \quad \chi^{23}=\frac{1}{B C} \chi_{23} .
\end{aligned}
$$

The WKB approximation can be utilized as [8]

$$
\chi_{v}=c_{v} \exp \left[\frac{i}{\hbar} S_{0}(t, r, \theta, \phi)+\Sigma \hbar^{n} S_{n}(t, r, \theta, \phi)\right],
$$

here, $c_{v}$ and $\left(S_{0}, S_{n}\right)$ are constant and arbitrary functions. 
In the WKB approximation the term $\hbar$ is considered just for the $1^{\text {st }}$ order and after neglecting the higher orders in the Lagrangian gravity Eq. (4), we get four set of equations

$$
\begin{aligned}
& A\left[c_{1}\left(\partial_{0} S_{0}\right)\left(\partial_{1} S_{0}\right)+\alpha c_{1}\left(\partial_{0} S_{0}\right)^{3}\left(\partial_{1} S_{0}\right)-c_{0}\left(\partial_{1} S_{0}\right)^{2}-\alpha c_{0}\left(\partial_{1} S_{0}\right)^{4}+c_{1} e A_{0}\left(\partial_{1} S_{0}\right)+c_{1} \alpha e A_{0}\left(\partial_{0} S_{0}\right)^{2}\left(\partial_{1} S_{0}\right)\right] \\
& +\frac{1}{B}\left[c_{2}\left(\partial_{0} S_{0}\right)\left(\partial_{2} S_{0}\right)+\alpha c_{2}\left(\partial_{0} S_{0}\right)^{3}\left(\partial_{2} S_{0}\right)-c_{0}\left(\partial_{2} S_{0}\right)^{2}-\alpha c_{0}\left(\partial_{2} S_{0}\right)^{4}+c_{2} e A_{0}\left(\partial_{2} S_{0}\right)+c_{2} \alpha e A_{0}\left(\partial_{0} S_{0}\right)^{2}\left(\partial_{2} S_{0}\right)\right] \\
& +\frac{1}{C}\left[c_{3}\left(\partial_{0} S_{0}\right)\left(\partial_{3} S_{0}\right)+\alpha c_{3}\left(\partial_{0} S_{0}\right)^{3}\left(\partial_{3} S_{0}\right)-c_{0}\left(\partial_{3} S_{0}\right)^{2}-\alpha c_{0}\left(\partial_{3} S_{0}\right)^{4}+c_{3} e A_{0}\left(\partial_{3} S_{0}\right)+c_{3} e A_{0}\left(\partial_{0} S_{0}\right)^{2}\left(\partial_{3} S_{0}\right)\right] \\
& -c_{0} m^{2}=0 \text {, } \\
& -\frac{1}{A}\left[c_{0}\left(\partial_{0} S_{0}\right)\left(\partial_{1} S_{0}\right)+\alpha c_{0}\left(\partial_{0} S_{0}\right)\left(\partial_{1} S_{0}\right)^{3}-c_{1}\left(\partial_{0} S_{0}\right)^{2}-\alpha c_{1}\left(\partial_{0} S_{0}\right)^{4}-c_{1} e A_{0}\left(\partial_{0} S_{0}\right)-\alpha c_{1} e A_{0}\left(\partial_{1} S_{0}\right)^{3}\right] \\
& +\frac{1}{B}\left[c_{2}\left(\partial_{1} S_{0}\right)\left(\partial_{2} S_{0}\right)+\alpha c_{2}\left(\partial_{1} S_{0}\right)^{3}\left(\partial_{2} S_{0}\right)-c_{1}\left(\partial_{2} S_{0}\right)^{2}-\alpha c_{1}\left(\partial_{2} S_{0}\right)^{4}\right]+\frac{1}{C}\left[c_{3}\left(\partial_{1} S_{0}\right)\left(\partial_{3} S_{0}\right)+\alpha c_{3}\left(\partial_{1} S_{0}\right)^{3}\left(\partial_{3} S_{0}\right)\right. \\
& \left.-c_{1}\left(\partial_{3} S_{0}\right)^{2}-\alpha c_{1}\left(\partial_{3} S_{0}\right)^{4}\right]-e A_{0}\left[c_{0}\left(\partial_{1} S_{0}\right)+\alpha c_{0}\left(\partial_{1} S_{0}\right)^{3}-c_{1}\left(\partial_{0} S_{0}\right)-\alpha c_{1}\left(\partial_{0} S_{0}\right)^{3}-e A_{0} c_{1}-\alpha c_{1} e A_{0}\left(\partial_{0} S_{0}\right)^{2}\right] \\
& -c_{1} m^{2}=0 \\
& -\frac{1}{A}\left[c_{0}\left(\partial_{0} S_{0}\right)\left(\partial_{2} S_{0}\right)+\alpha c_{0}\left(\partial_{0} S_{0}\right)\left(\partial_{2} S_{0}\right)^{3}-c_{2}\left(\partial_{0} S_{0}\right)^{2}-\alpha c_{2}\left(\partial_{0} S_{0}\right)^{4}-c_{2} e A_{0}\left(\partial_{0} S_{0}\right)-\alpha c_{2} e A_{0}\left(\partial_{0} S_{0}\right)^{3}\right] \\
& +A\left[c_{1}\left(\partial_{1} S_{0}\right)\left(\partial_{2} S_{0}\right)+\alpha c_{1}\left(\partial_{1} S_{0}\right)\left(\partial_{2} S_{0}\right)^{3}-c_{2}\left(\partial_{1} S_{0}\right)^{2}-\alpha c_{2}\left(\partial_{1} S_{0}\right)^{4}\right]+\frac{1}{C}\left[c_{3}\left(\partial_{2} S_{0}\right)\left(\partial_{3} S_{0}\right)+\alpha c_{3}\left(\partial_{2} S_{0}\right)^{3}\left(\partial_{3} S_{0}\right)\right. \\
& \left.-c_{2}\left(\partial_{3} S_{0}\right)^{2}-\alpha c_{2}\left(\partial_{3} S_{0}\right)^{4}\right]-\frac{e A_{0}}{A}\left[c_{0}\left(\partial_{2} S_{0}\right)+\alpha c_{0}\left(\partial_{2} S_{0}\right)^{3}-c_{2}\left(\partial_{0} S_{0}\right)-\alpha c_{2}\left(\partial_{0} S_{0}\right)^{3}-c_{2} e A_{0}-\alpha e A_{0}\left(\partial_{0} S_{0}\right)^{2}\right] \\
& +m^{2} c_{2}=0 \text {, } \\
& -\frac{1}{A}\left[c_{0}\left(\partial_{0} S_{0}\right)\left(\partial_{3} S_{0}\right)+\alpha c_{0}\left(\partial_{0} S_{0}\right)\left(\partial_{3} S_{0}\right)^{3}-c_{0}\left(\partial_{3} S_{0}\right)^{2}-c_{0}\left(\partial_{3} S_{0}\right)^{4}-e A_{0} c_{3}\left(\partial_{0} S_{0}\right)-\alpha c_{3} e A_{0}\left(\partial_{0} S_{0}\right)^{3}\right] \\
& -A\left[c_{1}\left(\partial_{1} S_{0}\right)\left(\partial_{3} S_{0}\right)+\alpha c_{1}\left(\partial_{1} S_{0}\right)\left(\partial_{3} S_{0}\right)^{3}-c_{3}\left(\partial_{1} S_{0}\right)^{2}-\alpha c_{3}\left(\partial_{1} S_{0}\right)^{4}\right]+\frac{1}{B}\left[c_{2}\left(\partial_{2} S_{0}\right)\left(\partial_{3} S_{0}\right)+\alpha c_{2}\left(\partial_{2} S_{0}\right)\left(\partial_{3} S_{0}\right)^{3}\right. \\
& \left.-c_{3}\left(\partial_{2} S_{0}\right)^{2}-\alpha c_{3}\left(\partial_{2} S_{0}\right)^{4}\right]-\frac{e A_{0}}{A}\left[c_{0}\left(\partial_{3} S_{0}\right)+\alpha c_{0}\left(\partial_{3} S_{0}\right)^{3}-c_{3}\left(\partial_{0} S_{0}\right)-\alpha c_{3}\left(\partial_{0} S_{0}\right)^{3}-c_{3} e A_{0}-\alpha e A_{0}\left(\partial_{0} S_{0}\right)^{2}\right] \\
& -m^{2} c_{3}=0 .
\end{aligned}
$$

Utilizing variables separation method, we consider

$$
S_{0}=-E_{0} t+W(r, \theta)+J \phi,
$$

where $E_{0}=(E-j \Omega), E$ and $J$ indicate the energy and angular momentum of particles corresponding with angle $\phi$. After utilizing the Eq. (12) into Eqs. (8)-(11), we get a four by four matrix as

$$
F\left(c_{0}, c_{1}, c_{2}, c_{3}\right)^{T}=0 .
$$


The above matrix seems to be non-trivial. Its components are devoted as follows

$$
\begin{aligned}
& F_{00}=-W_{r}^{2}-\alpha W_{r}^{4}-\frac{1}{A B}\left[W_{\theta}+\alpha W_{\theta}^{4}\right]-\frac{1}{A C}\left[\dot{J}^{2}+\alpha \dot{J}^{4}\right]-\frac{1}{A} m^{2}, \\
& F_{01}=-\left[E_{0}+\alpha E_{0}^{3}-e A_{0}\left(1+\alpha E_{0}^{2}\right)\right] W_{r}, \\
& F_{02}=-\frac{1}{A B}\left[E_{0}+\alpha E_{0}^{3}-e A_{0}\left(1+\alpha E_{0}^{2}\right)\right] W_{\theta}, \\
& F_{03}=-\frac{1}{A B}\left[E_{0}+\alpha E_{0}^{3}-e A_{0}\left(1+\alpha E_{0}^{2}\right)\right] \dot{j}, \\
& F_{10}=E_{0} W_{r}+\alpha E_{0}^{3} W_{r}^{4}-e A_{0}\left(W_{r}+\alpha W_{r}^{3}\right], \\
& F_{11}=E_{0}^{2}+\alpha E_{0}^{4}-e A_{0} E_{0}\left(1+\alpha W_{r}^{2}\right)-\frac{A}{B}\left(W_{\theta}^{2}+\alpha W_{\theta}^{4}\right)-\frac{A}{C}\left[\dot{J}^{2}+\alpha \dot{J}^{4}\right] \\
& \text { - } F m^{2}-e A_{0}\left[E_{0}+\alpha E_{0}-e A_{0}+e A_{0} \alpha W_{r}^{2}\right], \\
& F_{12}=\frac{A}{B}\left[W_{r}+\alpha W_{r}^{3}\right] W_{\theta}, \quad F_{13}=\frac{A}{C}\left[W_{r}+\alpha W_{r}^{3}\right] W_{\theta}, \\
& F_{20}=-\frac{1}{A B}\left[E_{0} W_{\theta}+\alpha E_{0} W_{\theta}^{3}\right]-\frac{e A_{0}}{A B}\left[W_{\theta}+\alpha W_{\theta}\right], \\
& F_{21}=\frac{A}{B}\left[W_{\theta}+\alpha W_{\theta}^{3}\right] W_{r} \\
& F_{22}=\frac{1}{A B}\left[E_{0}^{2}+\alpha E_{0}^{4}-e A_{0} E_{0}-\alpha e A_{0} E_{0}^{3}\right]-\frac{A}{B}\left[W_{r}^{2}+\alpha W_{r}^{4}\right]-\frac{1}{B C}\left[\dot{J}^{2}+\alpha \dot{j}^{4}\right] \\
& -\frac{1}{B} m^{2}-\frac{e A_{0}}{A B}\left[E_{0}+\alpha E_{0}^{3}-e A_{0}+\alpha e A_{0} E_{0}^{2}\right] \\
& F_{23}=\frac{1}{B C}\left[W_{r}+\alpha W_{r}^{3}\right] W_{\theta} \text {, } \\
& F_{30}=E_{0}\left[\dot{J}+\alpha \dot{j}^{3}\right]-\frac{e A_{0}}{A C}\left[\dot{J}+\alpha \dot{j}^{3}\right] \dot{J} \\
& F_{31}=-\frac{A}{C}\left[\dot{J}+\alpha \dot{j}^{3}\right] W_{r}, \quad F_{32}=\frac{1}{B C}\left[\dot{J}+\alpha \dot{j}^{3}\right] W_{\theta}, \\
& F_{33}=E_{0}^{2}+\alpha E_{0}^{4}-e A_{0} E_{0}-\alpha e A_{0} E_{0}^{3}+\frac{A}{C}\left[W_{r}^{2}+\alpha W_{r}^{4}\right]-\frac{1}{B C}\left[W_{\theta}^{2}+\alpha W_{\theta}^{4}\right] \\
& -\frac{1}{H} m^{2}-\frac{e A_{0}}{A C}\left[E_{0}+\alpha E_{0}^{3}-e A_{0}+\alpha e A_{0} W_{\theta}^{2}\right],
\end{aligned}
$$

where $\dot{J}=\partial_{\phi} S_{0}, W_{r}=\partial_{r} S_{0}$ and $W_{\theta}=\partial_{\theta} S_{0}$. In order to obtain the non-trivial matrix result, we put the determinant $\mathrm{F}$ equal to zero, so the action of imaginary part gets the form

$$
I m W^{ \pm}= \pm \operatorname{Im} \int \sqrt{\frac{\left(E_{0}-e A_{0}\right)^{2}+X_{1}\left[1+\alpha \frac{X_{2}}{X_{1}}\right]}{F^{2}}} d r
$$

where

$$
X_{1}=-\frac{F}{H} \dot{J}^{2}-F m^{2}, \quad X_{2}=E_{0}^{4}-F^{2} W_{r}^{4}-\frac{F}{H} \dot{J}^{4}-\left(e A_{0}\right)^{2} E_{0}^{2}-2 e A_{0} E_{0}^{3}
$$

The Eq. (13) implies

$$
\operatorname{ImW}^{ \pm}= \pm \pi \frac{\left(E_{0}-e A_{0}\right)}{2 \kappa\left(r_{+}\right)}[1+\alpha \Xi]
$$

here $\Xi=6\left(m^{2}+\frac{W_{\theta}^{2}+\dot{j}^{2} \csc ^{2} \theta}{r_{+}^{2}}\right)>0$ represents the kinetic energy of the emitted particles via tangent plane.

The modified tunneling rate for boson particles can be computed as

$$
\Gamma=\frac{\Gamma_{\text {emission }}}{\Gamma_{\text {absorption }}}=\exp \left[-4 \operatorname{Im} W^{+}\right]=\exp \left[-4 \pi \frac{\left(E_{0}-e A_{0}\right)}{2 \kappa\left(r_{+}\right)}\right][1+\alpha \Xi] .
$$


With the help of Boltzmann factor $\Gamma_{B}=\exp \left[\left(E-e A_{0} / T_{H}\right]\right.$, the Hawking temperature under the influence of quantum gravity for RN-DS $\mathrm{BH}$ with global monopole can be derived as

$$
T_{H}^{\prime}=\left[\frac{\frac{m}{r_{+}^{2}}-\frac{q^{2}}{r_{+}^{3}}-\frac{\Lambda}{3} r_{+}}{2 \pi}\right][1-\alpha \Xi] .
$$

For the massless boson particles case (i.e., $m=0$ ), Eq. (16) yields

$$
T_{H}^{\prime}=\left[\frac{-\frac{q^{2}}{r_{+}^{3}}-\frac{\Lambda}{3} r_{+}}{2 \pi}\right][1-\alpha \Xi] .
$$

The Hawking temperature for massive boson particle $(m \neq 0)$ is quite different from massless boson particle $(m=$ 0 ). For the massive boson particle, the Hawking temperature depends upon charge $q$, radius of outer horizon $r_{+}$, cosmological constant $\Lambda$ and quantum gravity parameter $\alpha$. We can observe that the corrected Hawking temperature does not only depend upon the $\mathrm{BH}$ properties but also depends upon the mass and angular momentum of the radiated particles and quantum corrections. The first order correction term is same as semiclassical original Hawking term, while the next order correction term must be smaller than the preceding term satisfying GUP.

The resulting Hawking temperature at which boson particle tunnel out through the RN-DS BH with global monopole horizon is like to the opposite temperature of boson particle at which they tunnel inward through the RN-DS BH with global monopole horizon $\left(T_{H}=-T_{H}\right)$. By neglecting the quantum gravity parameter $\alpha=0$, we get the temperature for RN-DS BH with global monopole [45]. For the case $\Lambda=0$, we obtain the modified temperature for RN BH [49]. Moreover, in the absence of gravity parameter (i.e., $\alpha=0$ ) as well as global monopole $\eta=0$, we recover the temperature for RN-DS BH [50]. It has worth to mention here that the global monopole does not affect the Hawking temperature. In the absence of charge $q=0$, global monopole $\eta=0$ as well as cosmological constant $\Lambda=0$, we get the modified temperature for Schwarzschild BH [51]. The above expression (16) reduces into Schwarzschild BH temperature for $q=0, \alpha=0, \Lambda=0$ which reads as [52].

\section{GRAPHICAL ANALYSIS OF TEMPERATURE}

We analyze the physical importance of these graphs in the presence of quantum gravity effects and examine the stable and unstable state of corresponding RN-DS BH with global monopole. We investigate the graphical behavior of temperature $T_{H}^{\prime}$ verses horizon $r_{+}$for the varying values of quantum gravity parameter $\alpha$ and charge $q$. The Hawking temperature highly increases with the decreasing horizon $r_{+}$and a small value of the parameter $\Xi=1$ can make a small change in temperature, only the non-physical case describes the instability condition of RN-DS $\mathrm{BH}$ with global monopole. The initial mass (larger than the remnant mass) gives three possible condition expresses physical significance relating on different values of horizon radius, charge and quantum gravity parameter.

- In FIG. 1, the corrected temperature increases with the increasing values of quantum gravity parameter and Hawking temperature decreases with the increasing radius horizon $r_{+}$(which is physical condition) and indicates the BH stability .

- In FIG. 2, the corrected temperature decreases gradually when, we increase the values of charge. The temperature exponentially decreases with the increasing horizon such that $T_{H}^{\prime} \rightarrow 0$ as $r_{+} \rightarrow \infty$. This asymptotically flat condition represents the physical and stable form of $\mathrm{BH}$.

FIG. 1 and 2 represents the totally stable form of BH. The increase in tunneling emission rate of RN-DS BH with global monopole with the increasing values of quantum gravity parameter is analyzed. According to Hawking's physical phenomenon (More emission of radiations reduces the size of $\mathrm{BH}$ radius). We observe this phenomenon in both plots, we observe maximum temperature at minimum value of horizon. According GUP condition the next order corrections must be small as compared to the standard term of Uncertainty relation. The positive temperature in these plots also satisfies the GUP relation, when GUP conditions does not satisfies temperature becomes negative (shows non-physical state of $\mathrm{BH}$ ). 


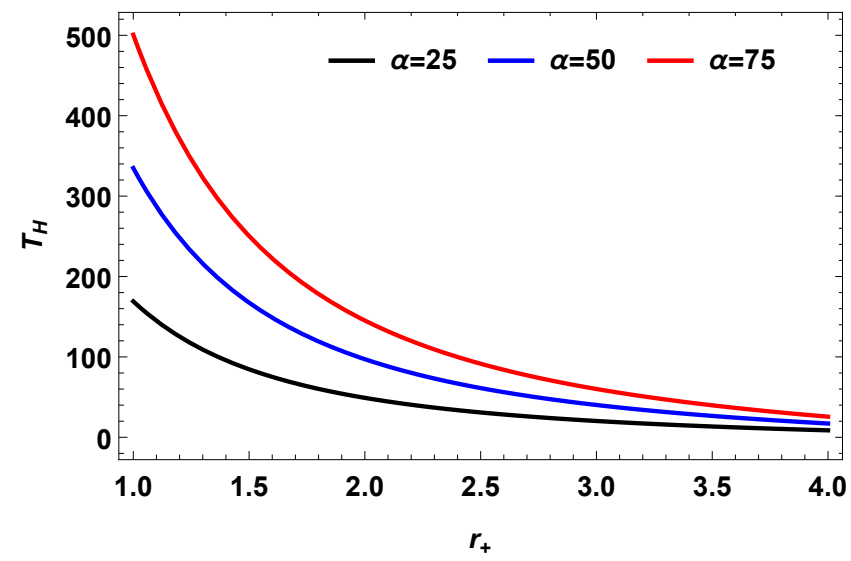

FIG. 1: $\mathrm{T}_{H}^{\prime}$ versus $r_{+}$for $m=30, q=3, \Lambda=0.5, \Xi=1$.

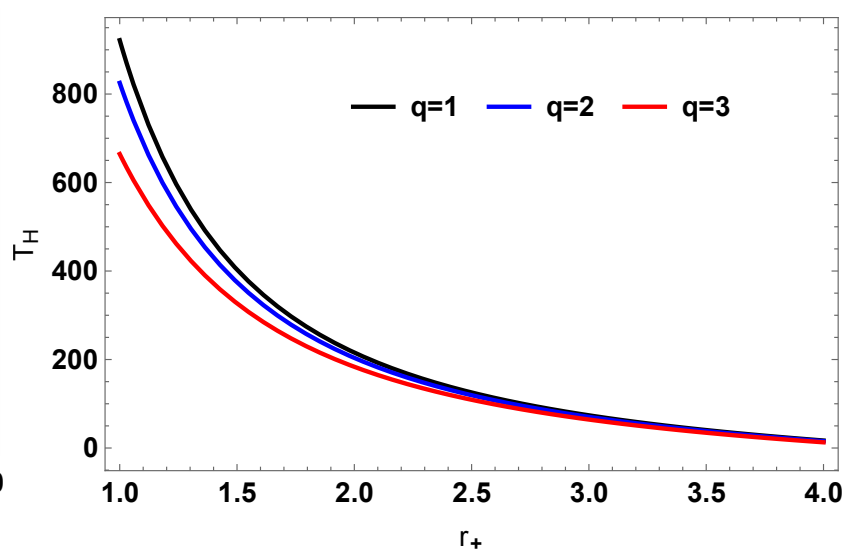

FIG. 2: $T_{H}^{\prime}$ versus $r_{+}$for $m=30, \Lambda=0.5, \alpha=100, \Xi=1$.

\section{5-DIMENSIONAL CHARGED BLACK HOLE}

This section provides the extension in Hawking temperature for 5D CBH. We assume a charged static spherically symmetric $(n+2)$ dimensional $\mathrm{BH}$ solution [31],

$$
d s^{2}=z(r) d t^{2}-\frac{1}{z(r)} d r^{2}-r^{2} d \Omega_{n}
$$

where $d \Omega_{n}$ is the unit $n$ sphere and $z(r)=1+\frac{Q^{2}}{r^{2 n-2}}-\frac{2 M}{r^{n}-1}$. For $n=2$ this reduce to $4 \mathrm{D}$ Reissner-Nordsrtöm (RN) metric while for $n=3$, we obtain a $5 \mathrm{D}$ charged $\mathrm{BH}$ solution as

$$
d s^{2}=\left(1-\frac{2 M}{r^{n}-1}+\frac{Q^{2}}{r^{2 n-2}}\right) d t^{2}-\left(1-\frac{2 M}{r^{n}-1}+\frac{Q^{2}}{r^{2 n-2}}\right)^{-1} d r^{2}-r^{2} d \theta^{2}-r^{2} \sin ^{2} \theta d \phi^{2}-r^{2} \sin ^{2} \theta \sin ^{2} \phi d \psi^{2}
$$

here, $Q$ and $M$ are the charge and mass of $\mathrm{BH}$ respectively.

The $\mathrm{BH}$ horizon can be found by solving for $r$ whose positive real roots will give horizon as follows

$$
r_{\text {outer }}=\sqrt{M+\sqrt{M^{2}-Q^{2}}}>0, \quad r_{\text {inner }}=\sqrt{M-\sqrt{M^{2}-Q^{2}}}>0,
$$

if $M^{2}>Q^{2}, r_{\text {outer }}>r_{\text {inner }}$ for $Q^{2}=M^{2}, r_{\text {outer }}=r_{\text {inner }}=\sqrt{M}$ (an extreme CBH) and for $Q^{2}>M^{2}$, both horizons disappear and naked singularity at $r=0$ and for $Q=0, r_{\text {outer }}=2 \sqrt{M}$ (4D Schwarzschild) and $r_{\text {inner }}=0$. This gives that like $4 \mathrm{D}$ case, the existence of charge is necessary for the existence of Cauchy horizon (inner horizon). The singularity of the 5D CBH can be seen in regions, $r_{\text {outer }}<r<\infty, 0<r<r_{\text {inner }}$ and $r_{\text {inner }}<r<r_{\text {outer }}$.

The line element from Eq.(19) takes the form

$$
d s^{2}=z(r) d t^{2}-\frac{1}{z(r)} d r^{2}-r^{2} d^{2} \theta-r^{2} \sin ^{2} \theta d \phi^{2}-r^{2} \sin ^{2} \theta \sin ^{2} \phi d \psi^{2} .
$$

The boson particles $\left(W^{ \pm}\right.$with spin equal to 1 ) are significant elements for the electro-weak interaction models, therefore the radiation of such particles should be more important in the investigation of Hawking temperature. There are various phenomena which are analyzed for the Hawking temperature because of a spectrum of tunneling across the horizon of 5D CBH. The tunneling process is associated on the fundamental of physical action particles which relates $\mathrm{CBH}$ radiation. The existence of positive and negative energy pairs of virtual boson particles is like the existence of anti-particle and particle pair production. Instantaneously, the positive and negative energy virtual boson particles are produced and annihilated in the form of particles pairs. The positive energy virtual boson particle evaporates by the tunneling through the horizon and emit as a part of Hawking radiation. The negative energy boson particles are immersed by the $\mathrm{CBH}$ or goes inside the $\mathrm{CBH}$. The law of conservation of energy and momentum are followed as in this method. The specific range of temperature at which the particles are observed stably and unstably from the horizon of 5D CBH. We have observed it for the 5D CBH (which is only charged) while we have proved it 
for more generalized 5D CBH (with quantum gravity and twisting parameters etc). We know that the determination still holds if background geometries of $\mathrm{BH}$ are more general.

By following the same procedure as Sec. II and utilizing Eq. (5) for the 5D CBH metric, we calculate the non zero values of $\chi^{\mu}$ and $\chi^{v \mu}$ as follows

$$
\begin{aligned}
& \chi^{0}=\frac{\chi_{0}}{z}, \quad \chi^{1}=-z \chi_{1}, \quad \chi^{2}=-\frac{1}{r^{2}} \chi_{2}, \quad \chi^{3}=-\frac{1}{r^{2} \sin ^{2} \theta} \chi_{3}, \quad \chi^{4}=-\frac{1}{r^{2} \sin ^{2} \theta \sin ^{2} \chi} \chi_{4}, \chi^{01}=-\chi_{01}, \\
& \chi^{03}=-\frac{1}{z r^{2} \sin ^{2} \theta} \chi_{03}, \quad \chi^{04}=-\frac{1}{z r^{2} \sin ^{2} \theta \sin ^{2} \chi} \chi_{04}, \quad \chi^{13}=\frac{z}{r^{2} \sin ^{2} \theta} \chi_{13}, \quad \chi^{14}=\frac{z}{r^{2} \sin ^{2} \theta \sin ^{2} \chi} \chi_{14}, \\
& \chi^{23}=\frac{1}{r^{4} \sin ^{2} \theta} \chi_{23}, \chi^{24}=\frac{1}{r^{4} \sin ^{2} \theta \sin ^{2} \chi} \chi_{24}, \quad \chi^{34}=\frac{z}{r^{4} \sin ^{4} \theta \sin ^{2} \chi} \chi_{34}, \quad \chi^{02}=-\frac{1}{z r^{2}} \phi_{02}, \quad \chi^{12}=\frac{z}{r^{2}} \chi_{12} .
\end{aligned}
$$

After putting the value of non-zero values of anti-symmetric tensor into Eq. (4) and using WKB approximation of Eq. (7), we get a set of 5 wave equations (for simplicity, we assume $A_{0}=A_{0}^{i}$ for all $i$ ) where $c_{v}$ is arbitrary constant, $\Theta_{0}$ and $\Theta_{n}$ are arbitrary functions.

Using the separation of variables strategy, we have

$$
\Theta_{0}=-\left(E-j_{1} \Omega_{1}-j_{2} \Omega_{2}\right) t+W(r)+\Theta(\theta, \phi)+J \psi,
$$

where $E$ is energy of particle and $\Omega$ is angular momentum. On the other hand, $\Theta$ and $J$ denotes the particles angular momenta associated to $\theta, \phi$ and $\psi$, respectively. For the above $\Theta_{0}$, the 4 set of Eqs. (29) to (33) can be written in terms of $5 \times 5$ matrix equation. The elements of the required matrix can be defined as

$$
H\left(c_{0}, c_{1}, c_{2}, c_{3}, c_{4}\right)^{T}=0 .
$$

The components of above matrix are given as are given as

$$
\begin{aligned}
H_{00}= & \left(\dot{W}^{2}+\alpha \dot{W}^{3}\right)+\frac{1}{z r^{2}}\left[\left(E-j_{1} \Omega_{1}-j_{2} \Omega_{2}\right)^{2}+\left(E-j_{1} \Omega_{1}-j_{2} \Omega_{2}\right)^{4}\right]+\frac{1}{z r^{2} \sin ^{2} \theta}\left[\Theta^{\prime 2}+\alpha \Theta^{4^{4}}\right] \\
& +\frac{1}{z r^{2} \sin ^{2} \theta \sin ^{2} \phi}\left[J^{2}+\alpha J^{4}\right]-\frac{m^{2}}{z}, \\
H_{01}= & {\left[\left(E-j_{1} \Omega_{1}-j_{2} \Omega_{2}\right)+\alpha\left(E-j_{1} \Omega_{1}-j_{2} \Omega_{2}\right)^{3}-e A_{0}-\left(E-j_{1} \Omega_{1}-j_{2} \Omega_{2}\right)^{2}\right] \dot{W}, } \\
H_{02}= & \frac{1}{z r^{2}}\left[\left(E-j_{1} \Omega_{1}-j_{2} \Omega_{2}\right)+\alpha\left(E-j_{1} \Omega_{1}-j_{2} \Omega_{2}\right)^{3}-e A_{0}-\alpha e A_{0}\left(E-j_{1} \Omega_{1}-j_{2} \Omega_{2}\right)\right] \dot{\Theta}, \\
H_{03}= & \frac{1}{z r^{2} \sin ^{2} \theta}\left[\left(E-j_{1} \Omega_{1}-j_{2} \Omega_{2}\right)+\alpha\left(E-j_{1} \Omega_{1}-j_{2} \Omega_{2}\right)^{3}-\alpha e A_{0}-\alpha e A_{0}\left(E-j_{1} \Omega_{1}-j_{2} \Omega_{2}\right)^{2}\right] \Theta^{\prime}, \\
H_{04}= & \frac{1}{z r^{2} \sin ^{2} \theta \sin ^{2} \phi}\left[\left(E-j_{1} \Omega_{1}-j_{2} \Omega_{2}\right)+\alpha\left(E-j_{1} \Omega_{1}-j_{2} \Omega_{2}\right)^{3}-e A_{0}-\alpha e A_{0}\left(E-j_{1} \Omega_{1}-j_{2} \Omega_{2}\right)^{2}\right] J, \\
H_{10}= & \left(E-j_{1} \Omega_{1}-j_{2} \Omega_{2}\right) \dot{W}+\alpha\left(E-j_{1} \Omega_{1}-j_{2} \Omega_{2}\right) \dot{W}^{3}-e A_{0}\left[\dot{W}+\alpha \dot{W}^{3}\right], \\
H_{11}= & \left(E-j_{1} \Omega_{1}-j_{2} \Omega_{2}\right)^{2}+\alpha\left(E-j_{1} \Omega_{1}-j_{2} \Omega_{2}\right)^{4}-e A_{0}\left(E-j_{1} \Omega_{1}-j_{2} \Omega_{2}\right)^{2}-e A_{0} \alpha\left(E-j_{1} \Omega_{1}-j_{2} \Omega_{2}\right)^{3}-\frac{z}{r^{2} \sin ^{2}} \\
& {\left[\dot{\Theta}^{2}+\alpha \dot{\Theta}^{4}\right]-\frac{1}{r^{2} \sin ^{2} \theta}\left[\Theta^{\prime 2}+\alpha \Theta^{4}\right]-\frac{z}{r^{2} \sin ^{2} \theta \sin ^{2} \phi}\left[J^{2}+\alpha J^{4}\right]+m^{2} z-e A_{0}\left[\left(E-j_{1} \Omega_{1}-j_{2} \Omega_{2}\right)+\alpha\right.} \\
& {\left[\left(E-j_{1} \Omega_{1}-j_{2} \Omega_{2}\right)^{3}-e A_{0}-\alpha e A_{0}\left(E-j_{1} \Omega_{1}-j_{2} \Omega_{2}\right)^{2}\right], } \\
H_{12}= & \frac{z}{r^{2}}\left[\dot{W}+\alpha \dot{W}^{3}\right] \dot{\Theta}, \\
H_{13}= & \frac{z}{r^{2} \sin ^{2} \theta}\left[\dot{W}+\alpha \dot{W}^{3}\right] \dot{\Theta}, \\
H_{14}= & \frac{z}{r^{2} \sin ^{2} \theta \sin ^{2} \phi}\left[\dot{W}+\alpha \dot{W}^{3}\right] J \\
H_{20}= & \frac{1}{z r^{2}}\left[\left(E-j_{1} \Omega_{1}-j_{2} \Omega_{2}\right) \dot{\Theta}+\alpha\left(E-j_{1} \Omega_{1}-j_{2} \Omega_{2}\right) \dot{\Theta}^{3}-\frac{e A_{0}}{z r^{2}}\left[\dot{\Theta}+\alpha \dot{\Theta}^{3}\right],\right. \\
H_{21}= & \frac{z}{r^{2}}\left[\dot{\Theta}+\alpha \dot{\Theta}^{3}\right] \dot{W},
\end{aligned}
$$




$$
\begin{aligned}
& H_{22}=\frac{1}{z r^{2}}\left[\left(E-j_{1} \Omega_{1}-j_{2} \Omega_{2}\right)^{2}+\alpha\left(E-j_{1} \Omega_{1}-j_{2} \Omega_{2}\right)^{4}-e A_{0}\left(E-j_{1} \Omega_{1}-j_{2} \Omega_{2}\right)-\alpha e A_{0}\left(E-j_{1} \Omega_{1}-j_{2} \Omega_{2}\right)^{3}\right] \\
& -\frac{z}{r^{2}}\left[\dot{W}^{2}+\alpha \dot{W}^{4}\right]-\frac{1}{r^{4} \sin ^{2} \theta}\left[\Theta^{\prime 2}+\alpha \Theta^{\prime 4}\right]-\frac{1}{r^{4} \sin ^{2} \theta \sin ^{2} \phi}\left[J^{2}+\alpha J^{4}\right]+\frac{m^{2}}{r^{2}}-\frac{e A_{0}}{z r^{2}}\left(E-j_{1} \Omega_{1}-j_{2} \Omega_{2}\right) \\
& \left.+\alpha\left(E-j_{1} \Omega_{1}-j_{2} \Omega_{2}\right)^{3}-e A_{0}-\alpha e A_{0}\left(E-j_{1} \Omega_{1}-j_{2} \Omega_{2}\right)^{2}\right], \\
& H_{23}=\frac{1}{-}\left[\dot{\Theta}+\alpha \dot{\Theta}^{2}\right] \Theta^{\prime}, \quad H_{24}=\frac{1}{r^{2} \sin ^{2} \theta \sin ^{2} \phi}\left[\dot{\Theta}+\alpha \dot{\Theta}^{2}\right] J, \\
& H_{30}=\frac{1}{z r^{2} \sin ^{2} \theta}\left[\left(E-j_{1} \Omega_{1}-j_{2} \Omega_{2}\right) \Theta^{\prime}+\alpha\left(E-j_{1} \Omega_{1}-j_{2} \Omega_{2}\right) \Theta^{\prime 3}-\frac{e A_{0}}{z r^{2} \sin ^{2} \theta}\left[\Theta^{\prime}+\alpha \Theta^{\prime}\right],\right. \\
& H_{31}=\frac{1}{r^{2} \sin ^{2} \theta}\left[\dot{\Theta}^{0}+\alpha \dot{\Theta}^{\prime 3}\right] \dot{W}, \\
& H_{32}=\frac{1}{r^{4} \sin ^{2} \theta}\left[\Theta^{\prime}+\alpha \Theta^{\prime 3}\right] \dot{\Theta}, \\
& H_{33}=\frac{1}{z r^{2} \sin ^{2} \theta}\left[\left(E-j_{1} \Omega_{1}-j_{2} \Omega_{2}\right)^{2}+\alpha\left(E-j_{1} \Omega_{1}-j_{2} \Omega_{2}\right)^{4}\right]-\frac{1}{r^{2} \sin ^{2} \theta}\left[\dot{W}^{2}+\alpha \dot{W}^{4}\right]-\frac{1}{r^{4} \sin ^{2} \theta}\left[\dot{\Theta}^{2}+\alpha \dot{\Theta}^{4}\right] \\
& -\frac{1}{r^{4} \sin ^{4} \theta \sin ^{2} \phi}\left[J^{2}+\alpha J^{4}\right]+\frac{m^{2}}{r^{2} \sin ^{2} \theta}-\frac{e A_{0}}{z r^{2} \sin ^{2} \theta}\left[\left(E-j_{1} \Omega_{1}-j_{2} \Omega_{2}\right)+\alpha\left(E-j_{1} \Omega_{1}-j_{2} \Omega_{2}\right)^{3}\right]-e A_{0} \\
& \left.-\alpha A_{0}\left(E-j_{1} \Omega_{1}-j_{2} \Omega_{2}\right)^{2}\right], \\
& H_{34}=\frac{1}{r^{4} \sin ^{4} \theta \sin ^{2} \theta}\left[\Theta^{\prime}+\alpha \Theta^{\beta^{3}}\right] J, \\
& H_{40}=\frac{1}{z r^{2} \sin ^{2} \theta \sin ^{2} \phi}\left[\left(E-j_{1} \Omega_{1}-j_{2} \Omega_{2}\right) J+\alpha\left(E-j_{1} \Omega_{1}-j_{2} \Omega_{2}\right) J^{3}-\frac{e A_{0}}{z r^{2} \sin ^{2} \theta \sin ^{2} \phi}\left[J+\alpha J^{3}\right],\right. \\
& H_{41}=\frac{z}{r^{2} \sin ^{2} \theta \sin ^{2} \phi}\left[\left(E-j_{1} \Omega_{1}-j_{2} \Omega_{2}\right) J+\alpha\left(E-j_{1} \Omega_{1}-j_{2} \Omega_{2}\right)^{2} J^{3}\right]-\frac{e A_{0}}{r^{2} \sin ^{2} \theta \sin ^{2} \phi}\left[J+\alpha J^{3}\right], \\
& H_{42}=\frac{z}{r^{4} \sin ^{2} \theta \sin ^{2} \phi}\left[J+\alpha J^{3}\right] \dot{\Theta}, \\
& H_{43}=\frac{z}{r^{4} \sin ^{4} \theta \sin ^{2} \phi}\left[J+\alpha J^{3}\right] \Theta^{\prime}, \\
& H_{44}=\frac{1}{z r^{2} \sin ^{2} \theta \sin ^{2} \phi}\left[\left(E-j_{1} \Omega_{1}-j_{2} \Omega_{2}\right)^{2}+\alpha\left(E-j_{1} \Omega_{1}-j_{2} \Omega_{2}\right)^{4}-e A_{0}\left(E-j_{1} \Omega_{1}-j_{2} \Omega_{2}\right)\right. \\
& \left.-\alpha e A_{0}\left(E-j_{1} \Omega_{1}-j_{2} \Omega_{2}\right)^{3}\right]-\frac{z}{r^{2} \sin ^{2} \theta \sin ^{2} \phi}\left[\dot{W}^{2}+\alpha \dot{W}^{4}\right]-\frac{1}{r^{4} \sin ^{2} \theta \sin ^{2} \phi}\left[\dot{\Theta}^{2}+\alpha \dot{\Theta}^{4}\right] \\
& -\frac{1}{r^{4} \sin ^{2} \theta \sin ^{2} \phi}\left[\Theta^{\prime^{2}}+\alpha \Theta^{\prime^{4}}\right]+\frac{m^{2}}{r^{2} \sin ^{2} \theta \sin ^{2} \phi}-\frac{e A_{0}}{z r^{2} \sin ^{2} \theta \sin ^{2} \phi} \\
& {\left[\left(E-j_{1} \Omega_{1}-j_{2} \Omega_{2}\right)+\alpha\left(E-j_{1} \Omega_{1}-j_{2} \Omega_{2}\right)^{3}-e A_{0}-\alpha e A_{0}\left(E-j_{1} \Omega_{1}-j_{2} \Omega_{2}\right)^{2}\right],}
\end{aligned}
$$

where $\dot{W}=\partial_{r} \Theta_{0}, \dot{\Theta}=\partial_{\theta} \Theta_{0}$ and $\Theta^{\prime}=\partial_{\phi} \Theta_{0}$. In order to find a non-trivial solution, we set $|\mathbf{H}|=0$ and obtain

$$
\operatorname{Im} W^{ \pm}= \pm \int \sqrt{\frac{\left(E-j_{1} \Omega_{1}-j_{2} \Omega_{2}-e A_{0}\right)^{2}+X_{1}\left[1+\alpha \frac{X_{2}}{X_{1}}\right]}{z^{2}}} d r= \pm i \pi \frac{\left(E-j_{1} \Omega_{1}-j_{2} \Omega_{2}-e A_{0}\right)+[1+\Xi \alpha]}{2 \kappa\left(r_{+}\right)},
$$

where

$$
\begin{aligned}
X_{1} & =-\frac{\Theta^{\prime^{2}}}{z \sin ^{2} \theta}-\frac{1}{z r^{2} \sin ^{2} \theta \sin ^{2} \phi}+\frac{m^{2}}{z}, \\
X_{2} & =\frac{1}{z^{2}}\left[\left(E-j_{1} \Omega_{1}-j_{2} \Omega_{2}\right)^{4}+\left(e A_{0}\right)^{2}\left(E-j_{1} \Omega_{1}-j_{2} \Omega_{2}\right)^{2}-2 e A_{0}\left(E-j_{1} \Omega_{1}-j_{2} \Omega_{2}\right)-\dot{W}^{4}\right. \\
& -\frac{\Theta^{\prime^{4}}}{z \sin ^{2} \theta}-\frac{J^{4}}{z r^{2} \sin ^{2} \theta \sin ^{2} \phi} .
\end{aligned}
$$


The BH surface gravity is given by

$$
\kappa\left(r_{+}\right)=\frac{4 M}{r_{+}^{3}}-\frac{4 Q^{2}}{r_{+}^{5}} .
$$

The required boson tunneling probability can be expressed as

$$
\Gamma=\frac{\Gamma_{\text {emission }}}{\Gamma_{\text {absortion }}}=e^{-4 I m W^{+}}=\exp \left[-4 \pi \frac{\left(E-j_{1} \Omega_{1}-j_{2} \Omega_{2}-e A_{0}\right)}{\frac{4 M}{r_{+}^{3}}-\frac{4 Q^{2}}{r_{+}^{5}}}\right][1+\Xi \alpha] .
$$

The corrected temperature in this case is given by

$$
T_{H}=\frac{\frac{M}{r_{+}^{3}}-\frac{Q^{2}}{r_{+}}}{\pi}[1-\Xi \alpha] .
$$

The Hawking temperature depends upon the mass, charge and radial coordinate $r_{+}$of $\mathrm{BH}$. If $\alpha$ is zero then the temperature will be independent of quantum gravity.

Stopping of the $\mathrm{BH}$ evaporation will be achieved in the case of quantum corrections. The calculations of quantum corrections slow down the increase in Hawking temperature during the radiation process. By continuing this procedure, there is a balance state. At this state, the evaporation stops and remnants are produced. It has been worth to note that if $(\alpha=0)$, we have obtained the original Hawking temperature for $5 D \mathrm{BH}$ in the absence of quantum gravity parameter. To estimate the residual masses of the BHs at the level of the order of the magnitude, for $Q=0$, the obtained temperature reduces to the case of Schwarzschild BH, i.e.,

$$
T_{H}^{\prime}=\frac{1}{8 \pi M}\left[1-6 \alpha\left(m^{2}+\frac{\left(J_{\theta}^{2}+J_{\phi}^{2} \csc ^{2} \theta\right)}{r_{+}^{2}}\right)\right]
$$

where $\left(m^{2}+\frac{\left(J_{\theta}^{2}+J_{\phi}^{2} \csc ^{2} \theta\right)}{r_{+}^{2}}\right)$ denotes the kinetic energy component of radiated particles related with tangent plane at horizon. For residual mass, we approximate the kinetic energy component as $E^{2}$. Quantum corrections decelerate the increase in temperature during the radiation process. These corrections cause the radiation ceased at some specific temperature, leaving the remnant mass. The temperature stops increasing when this condition holds

$$
(M-d M)(1+\alpha \Xi) \simeq M .
$$

For $d M=E, \alpha=\frac{\alpha_{0}}{M_{p}^{2}}$ and $E \simeq M_{p}$, we get the following relation

$$
M_{\text {Res }} \simeq \frac{M_{p}^{2}}{\alpha_{0} E} \gtrsim \frac{M_{p}}{\alpha_{0}}, \quad T_{\text {Res }} \lesssim \frac{\alpha_{0}}{8 \pi M_{p}} .
$$

\section{GRAPHICAL ANALYSIS}

We analyze the graphical expression of generalized temperature $T_{H}^{\prime}$ versus outer horizon $\left(r_{+}\right)$as indicated in FIG. 3 and FIG. 4, respectively and for our result, we fix the arbitrary parameter $\Xi=1$ and mass $M=1000$.

- In the FIG. 3, the quantum gravity is fixed $\alpha=100$ and the charge varies for different values in the range $\left(0 \leq r_{+} \leq 5\right)$. We can observe that the temperature of $\mathrm{BH}$ decreases as we increase the values of charge.

- In the FIG. 4, the charge is fixed $Q=25$ and the quantum gravity varies for different values in the range $\left(0 \leq r_{+} \leq 5\right)$. We can observe in plot, the temperature decreases as we increase the values of $\alpha$.

From both plots, we conclude that, initially, the $\mathrm{CBH}$ is unstable but as the time goes on the $\mathrm{CBH}$ becomes stable and after a maximum height at very high temperature, it drops down and gets an asymptotically flat condition till $r_{+} \longrightarrow \infty$. This condition with positive temperature guarantees the $\mathrm{BH}$ stable condition and shows its physical form. The negative temperature shows the unstable condition of $\mathrm{CBH}$ because at this point GUP condition does not satisfy.

- FIG. 5 indicates the behavior of temperature in the absence of quantum gravity parameter $\alpha=0$. We can observe that the value of temperature is smaller than in the presence of correction parameter as compared in the absence of correction parameter. One can also observe thatin the absence of gravity parameter the decreases with the increasing value of horizon and gets an asymptotically flat condition to ensure the $\mathrm{BH}$ stability. 

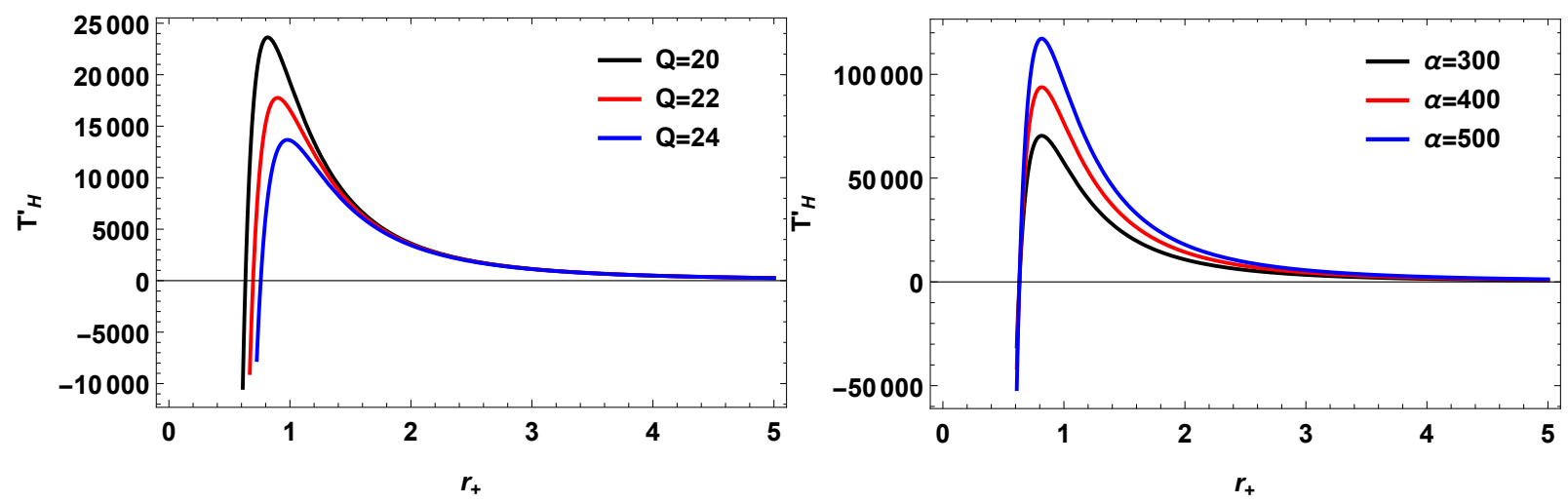

FIG. 3: Hawking temperature $T_{H}^{\prime}$ versus outer horizon ra-FIG. 4: Hawking temperature $T_{H}^{\prime}$ versus outer horizon radius $r_{+}$for $M=1000, \alpha=100$ and $\Xi=1$. dius $r_{+}$for $M=1000, Q=25$ and $\Xi=1$.

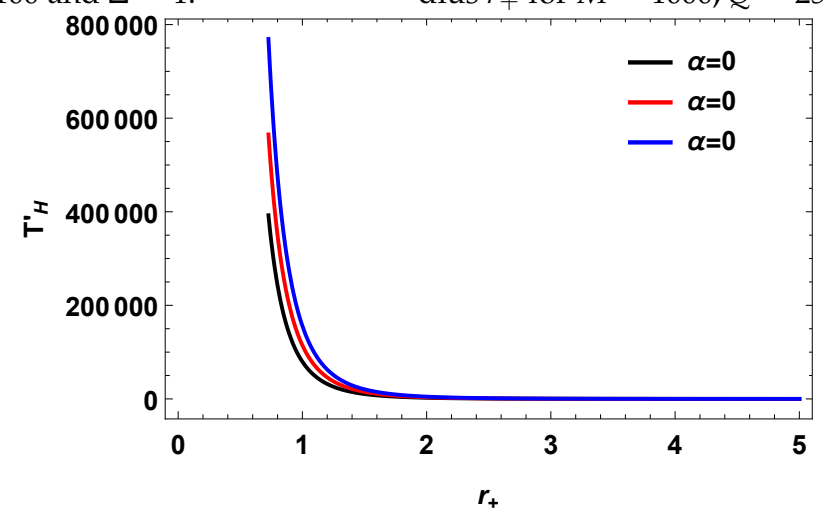

FIG. 5: $T_{H}^{\prime}$ versus outer horizon radius $r_{+}$for $\alpha=0$.

\section{SUMMARY AND DISCUSSION}

This paper provide an extension for Hawking temperature of RN-DS BH with global monopole as well as 5D CBH under the effects of quantum gravity. In this work, we have analyzed the quantum tunneling method of massive as well as massless boson particles from an electrically charged RN-DS BH with global monopole. Firstly, the quantum tunneling approach has been used with $(t, r, \theta, \phi)$ type of coordinate system to study the equation of motion of boson particles with GUP. For this investigation, we have applied the Hamilton-Jacobi ansatz method and the WKB approximation for massive charged spin-1 particles (bosons). The boson particle tunneling and temperature depends upon the geometry of $\mathrm{BH}$. In our phenomenon, we have modified the Lagrangian gravity equation in curved spacetime. We have analyzed the semiclassical action in the power series of the Planck's constant to compute the Modified tunneling rate of the vector particles. Subsequently, the quantum surface gravity has been computed and the back reaction effects of the space-time has also been neglected by suitable reason of this method. We concluded that the modified tunneling probability are not just dependent on the BHs properties, but also on the properties of emitted vector bosons (i.e., particle charge, energy, surface gravity, potential and total angular momentum). Finally, the quantum corrected Hawking temperature for corresponding $\mathrm{BH}$ has been derived. Furthermore, it is important to note that the modified tunneling probability as well as the modified Hawking temperature depend on the quantum particles, which contribute gravitational radiation in form of massive particle (BH's energy carrier) tunneling. It is examined that the tunneling rate and Hawking temperature depend on the RN-DS BH with global monopole geometry. The modified Hawking temperature depends upon charge $q$, radius of outer horizon $r_{+}$, cosmological constant $\Lambda$ and quantum gravity parameter $\alpha$. It is important to mention here that, by neglecting the quantum gravity parameter $\alpha=0$, we obtain the temperature for RN-DS BH with global monopole [45]. For the case $\Lambda=0$, we obtain the modified temperature for RN BH [49]. Moreover, in the absence of gravity parameter $\alpha=0$ as well as global monopole $\eta=0$, we recover the temperature for RN-DS BH [50]. It has worth to mention here that the global monopole does not affect the Hawking temperature. In the absence of charge $q=0$, global monopole $\eta=0$ as well as cosmological constant $\Lambda=0$, we get the modified temperature for Schwarzschild BH [51]. The above expression (16) reduces into Schwarzschild BH temperature for $q=0, \alpha=0, \Lambda=0$ which reads as [52]. Our calculations are 
similar to the statement that the tunneling radiation is independent of the type of the particles and this result is also hold for different coordinate frames by applying coordinate transformations.

Furthermore, we have investigated the physical significance of our plots. The graphical interpretation of corrected temperature $T_{H}^{\prime}$ verses horizon $r_{+}$for different values of gravity parameter $\alpha$ and charge $q$ depicts the stable and unstable form of BH. From our plots, we conclude that the tunneling emission rate increases (gives high temperature) with the increasing values of $\alpha$. The corrected temperature of RN-DS BH satisfies the both GUP and Hawking's conditions, that guarantee the physical and stable states of BH. According to Hawking's physical phenomenon (More emission of radiations reduces the size of $\mathrm{BH}$ radius). We observe this phenomenon in both plots, we observe maximum temperature at minimum value of horizon. According GUP condition the next order corrections must be small as compared to the standard term of Uncertainty relation. The positive temperature in these plots also satisfies the GUP relation, when GUP conditions does not satisfies temperature becomes negative (shows non-physical state of $\mathrm{BH})$.

Moreover, we have analyzed the radiation spectrum of boson particles from 5D CBH. By following the same process, we have calculated the corrected temperature for $\mathrm{CBH}$ and the corrected Hawking temperature depend on $\mathrm{BH}$ mass, charge and radius of horizon, as well as on the quantum gravity parameter of the emitted boson particles. We have analyzed physical and non-physical behavior of $\mathrm{BH}$ temperature for charge and gravity, respectively. We have analyzed the behavior of temperature verses $r_{+}$, for the particular ranges, the temperature decreases with the increase of gravity parameter. When the charge was fixed and quantum gravity changes from 300 to 500, as well as quantum gravity was fixed and charge change from 20 to 24 , we observed that the $\mathrm{BH}$ will be stable in the approximation range $0 \leq r+\leq 5$ after getting a maximum height at very high temperature. The unstable condition of $\mathrm{BH}$ appears at negative temperature, where GUP condition does not satisfy. When the quantum gravity effects are ignored, we have found the absolute Hawking temperature of 5D CBH. Moreover, for charge free case, the temperature and its correction correspond to the condition of Schwarzschild BH.

\section{APPENDIX}

The set of field equations is given as follows

$$
\begin{aligned}
& -\left[c_{1}\left(\partial_{1} \Theta_{0}\right)\left(\partial_{0} \Theta_{0}\right)+c_{1}\left(\partial_{1} \Theta_{0}\right)\left(\partial_{0} \Theta_{0}\right)^{3} \alpha-c_{0}\left(\partial_{1} \Theta_{0}\right)^{2}-\left(\partial_{1} \Theta_{0}\right)^{4} \alpha c_{0}+\left(\partial_{1} \Theta_{0}\right) c_{1} e A_{0}+c_{1}\left(\partial_{1} \Theta_{0}\right)\left(\partial_{0} \Theta_{0}\right)^{2} e A_{0}\right] \\
& -\frac{1}{z r^{2}}\left[c_{2}\left(\partial_{2} \Theta_{0}\right)\left(\partial_{0} \Theta_{0}\right)+\alpha c_{2}\left(\partial_{2} \Theta_{0}\right)\left(\partial_{0} \Theta_{0}\right)^{3}-c_{0}\left(\partial_{2} \Theta_{0}\right)^{2}-c_{0}\left(\partial_{0} \Theta_{0}\right)^{4} \alpha+c_{2}\left(\partial_{2} \Theta_{0}\right) e A_{0}+c_{2}\left(\partial_{0} \Theta_{0}\right)^{2}\left(\partial_{2} \Theta_{0}\right) \alpha e A_{0}\right] \\
& -\frac{1}{z r^{2} \sin ^{2} \theta}\left[c_{3}\left(\partial_{3} \Theta_{0}\right)\left(\partial_{0} \Theta_{0}\right)+c_{3}\left(\partial_{3} \Theta_{0}\right)\left(\partial_{0} \Theta_{0}\right)^{3} \alpha-c_{0}\left(\partial_{3} \Theta_{0}\right)^{2}-c_{0}\left(\partial_{3} \Theta_{0}\right)^{4} \alpha+c_{3}\left(\partial_{3} \Theta_{0}\right) e A_{0}+c_{3}\left(\partial_{3} \Theta_{0}\right)\left(\partial_{0} \Theta_{0}\right)^{2} e A_{0}\right] \\
& -\frac{1}{z r^{2} \sin ^{2} \theta \sin ^{2} \phi}\left[c_{4}\left(\partial_{4} \Theta_{0}\right)\left(\partial_{0} \Theta_{0}\right)+c_{4}\left(\partial_{4} \Theta_{0}\right)\left(\partial_{0} \Theta_{0}\right)^{3} \alpha-c_{0}\left(\partial_{4} \Theta_{0}\right)^{2}-c_{0}\left(\partial_{4} \Theta_{0}\right)^{4} \alpha+c_{4}\left(\partial_{4} \Theta_{0}\right) e A_{0}+c_{4} e A_{0} \alpha\right. \\
& \left.\left(\partial_{4} \Theta_{0}\right)\left(\partial_{0} \Theta_{0}\right)^{2}\right]-\frac{m^{2}}{z} c_{0}=0, \\
& -\left[c_{0}\left(\partial_{1} \Theta_{0}\right)\left(\partial_{0} \Theta_{0}\right)+c_{0}\left(\partial_{1} \Theta_{0}\right)^{3}\left(\partial_{0} \Theta_{0}\right) \alpha-c_{1}\left(\partial_{0} \Theta_{0}\right)^{2}-c_{1}\left(\partial_{0} \Theta_{0}\right)^{4} \alpha-c_{1}\left(\partial_{0} \Theta_{0}\right) e A_{0}-c_{1}\left(\partial_{1} \Theta_{0}\right)^{3} e A_{0} \alpha\right]+\frac{z}{r^{2}} \\
& {\left[c_{2}\left(\partial_{2} \Theta_{0}\right)\left(\partial_{1} \Theta_{0}\right)+c_{2}\left(\partial_{2} \Theta_{0}\right)\left(\partial_{1} \Theta_{0}\right)^{3} \alpha-c_{1}\left(\partial_{2} \Theta_{0}\right)^{2}-c_{1}\left(\partial_{2} \Theta_{0}\right)^{4} \alpha\right]+\frac{z}{r^{2} \sin ^{2} \theta}\left[c_{3}\left(\partial_{3} \Theta_{0}\right)\left(\partial_{1} \Theta_{0}\right)+c_{3}\left(\partial_{3} \Theta_{0}\right)\left(\partial_{1} \Theta_{0}\right)^{3} \alpha\right.} \\
& \left.-c_{1}\left(\partial_{3} \Theta_{0}\right)^{2}-c_{1}\left(\partial_{3} \Theta_{0}\right)^{4} \alpha\right]+\frac{z}{r^{2} \sin ^{2} \theta \sin ^{2} \phi}\left[c_{4}\left(\partial_{4} \Theta_{0}\right)\left(\partial_{1} \Theta_{0}\right)+c_{4}\left(\partial_{4} \Theta_{0}\right)\left(\partial_{1} \Theta_{0}\right)^{3} \alpha-c_{1}\left(\partial_{4} \Theta_{0}\right)^{2}-c_{1}\left(\partial_{4} \Theta_{0}\right)^{4} \alpha\right] \\
& \left.+m^{2} z c_{1}-\left[c_{0}\left(\partial_{1} \Theta_{0}\right)+c_{0}\left(\partial_{1} \Theta_{0}\right)^{3} \alpha-c_{1}\left(\partial_{0} \Theta_{0}\right)-c_{1}\left(\partial_{0} \Theta_{0}\right)^{3} \alpha-c_{1} e A_{0}-c_{1}\left(\partial_{0} \Theta_{0}\right)^{2}\right) e A_{0} \alpha\right] e A_{0}=0, \\
& -\frac{1}{z r^{2}}\left[c_{0}\left(\partial_{2} \Theta_{0}\right)\left(\partial_{0} \Theta_{0}\right)+c_{0}\left(\partial_{2} \Theta_{0}\right)^{3}\left(\partial_{0} \Theta_{0}\right) \alpha-c_{2}\left(\partial_{0} \Theta_{0}\right)^{2}-c_{2}\left(\partial_{0} \Theta_{0}\right)^{4} \alpha-c_{2}\left(\partial_{0} \Theta_{0}\right) e A_{0}-c_{2} e A_{0}\left(\partial_{0} \Theta_{0}\right)^{3} \alpha\right]+\frac{z}{r^{2}} \\
& {\left[c_{1}\left(\partial_{2} \Theta_{0}\right)\left(\partial_{1} \Theta_{0}\right)+c_{1}\left(\partial_{2} \Theta_{0}\right)^{3}\left(\partial_{1} \Theta_{0}\right) \alpha-c_{2}\left(\partial_{1} \Theta_{0}\right)^{2}-c_{2}\left(\partial_{1} \Theta_{0}\right)^{4} \alpha\right]+\frac{1}{r^{4} \sin ^{2} \theta}\left[c_{3}\left(\partial_{3} \Theta_{0}\right)\left(\partial_{2} \Theta_{0}\right)+c_{3}\left(\partial_{3} \Theta_{0}\right)\left(\partial_{2} \Theta_{0}\right)^{3} \alpha\right.} \\
& \left.-c_{2}\left(\partial_{3} \Theta_{0}\right)^{2}-c_{2}\left(\partial_{3} \Theta_{0}\right)^{4} \alpha\right]+\frac{1}{r^{4} \sin ^{2} \theta \sin ^{2} \phi}\left[c_{4}\left(\partial_{4} \Theta_{0}\right)\left(\partial_{2} \Theta_{0}\right)+c_{4}\left(\partial_{4} \Theta_{0}\right)\left(\partial_{2} \Theta_{0}\right)^{3} \alpha-c_{2}\left(\partial_{4} \Theta_{0}\right)^{2}-c_{2}\left(\partial_{4} \Theta_{0}\right)^{4} \alpha\right] \\
& +\frac{m^{2} c_{2}}{r^{2}}-\frac{e A_{0}}{z r^{2}}\left[c_{0}\left(\partial_{2} \Theta_{0}\right)+c_{0}\left(\partial_{2} \Theta_{0}\right)^{3} \alpha-c_{2}\left(\partial_{0} \Theta_{0}\right)-c_{2}\left(\partial_{0} \Theta_{0}\right)^{3} \alpha-c_{2} e A_{0}-c_{2}\left(\partial_{0} \Theta_{0}\right)^{2} \alpha e A_{0}\right]=0,
\end{aligned}
$$




$$
\begin{aligned}
& -\frac{1}{z r^{2} \sin ^{2} \theta}\left[c_{0}\left(\partial_{3} \Theta_{0}\right)\left(\partial_{0} \Theta_{0}\right)+c_{0}\left(\partial_{3} \Theta_{0}\right)^{3}\left(\partial_{0} \Theta_{0}\right) \alpha-c_{0}\left(\partial_{3} \Theta_{0}\right)^{2}-c_{0}\left(\partial_{3} \Theta_{0}\right)^{4} \alpha-c_{3}\left(\partial_{0} \Theta_{0}\right) e A_{0}-c_{3}\left(\partial_{0} \Theta_{0}\right)^{3} \alpha e A_{0}\right] \\
& +\frac{1}{r^{2} \sin ^{2} \theta}\left[c_{1}\left(\partial_{3} \Theta_{0}\right)\left(\partial_{1} \Theta_{0}\right)+c_{1}\left(\partial_{3} \Theta_{0}\right)^{3}\left(\partial_{1} \Theta_{0}\right) \alpha-c_{3}\left(\partial_{1} \Theta_{0}\right)^{2}-c_{3}\left(\partial_{1} \Theta_{0}\right)^{4} \alpha\right]+\frac{1}{r^{4} \sin ^{2} \theta}\left[c_{2}\left(\partial_{3} \Theta_{0}\right)\left(\partial_{2} \Theta_{0}\right)+c_{2} \alpha\right. \\
& \left.\left(\partial_{3} \Theta_{0}\right)^{3}\left(\partial_{2} \Theta_{0}\right)-c_{3}\left(\partial_{2} \Theta_{0}\right)^{2}-c_{3}\left(\partial_{2} \Theta_{0}\right)^{4} \alpha\right]+\frac{1}{r^{4} \sin ^{4} \theta \sin ^{2} \phi}\left[c_{4}\left(\partial_{4} \Theta_{0}\right)\left(\partial_{3} \Theta_{0}\right)+c_{4}\left(\partial_{4} \Theta_{0}\right)\left(\partial_{3} \Theta_{0}\right)^{3} \alpha-c_{3}\left(\partial_{4} \Theta_{0}\right)^{2}\right. \\
& \left.-c_{3}\left(\partial_{4} \Theta_{0}\right)^{4} \alpha\right]+\frac{m^{2} c_{3}}{r^{2} \sin ^{2} \theta}-\frac{e A_{0}}{z r^{2} \sin ^{2} \theta}\left[c_{0}\left(\partial_{3} \Theta_{0}\right)+c_{0}\left(\partial_{3} \Theta_{0}\right)^{3} \alpha-c_{3}\left(\partial_{0} \Theta_{0}\right)-c_{3}\left(\partial_{0} \Theta_{0}\right)^{3} \alpha-c_{3} e A_{0}-c_{3}\left(\partial_{0} \Theta_{0}\right)^{2} \alpha e A_{0}\right] \\
& =0 \text {, } \\
& -\frac{1}{z r^{2} \sin ^{2} \theta \sin ^{2} \phi}\left[c_{0}\left(\partial_{4} \Theta_{0}\right)\left(\partial_{0} \Theta_{0}\right)+c_{0}\left(\partial_{4} \Theta_{0}\right)^{3}\left(\partial_{0} \Theta_{0}\right) \alpha-c_{4}\left(\partial_{0} \Theta_{0}\right)^{2}-c_{4}\left(\partial_{0} \Theta_{0}\right)^{4} \alpha-c_{4}\left(\partial_{0} \Theta_{0}\right) e A_{0}-c_{4}\left(\partial_{0} \Theta_{0}\right)^{3} e A_{0} \alpha\right] \\
& +\frac{z}{r^{4} \sin ^{2} \theta \sin ^{2} \phi}\left[c_{1}\left(\partial_{4} \Theta_{0}\right)\left(\partial_{1} \Theta_{0}\right)+c_{1}\left(\partial_{4} \Theta_{0}\right)^{3}\left(\partial_{1} \Theta_{0}\right) \alpha-c_{4}\left(\partial_{1} \Theta_{0}\right)^{2}-c_{4}\left(\partial_{1} \Theta_{0}\right)^{4} \alpha\right]+\frac{1}{r^{4} \sin ^{2} \theta \sin ^{2} \phi} \\
& {\left[c_{2}\left(\partial_{4} \Theta_{0}\right)\left(\partial_{2} \Theta_{0}\right)+c_{4}\left(\partial_{4} \Theta_{0}\right)^{3}\left(\partial_{2} \Theta_{0}\right) \alpha-c_{4}\left(\partial_{2} \Theta_{0}\right)^{2}-c_{4}\left(\partial_{2} \Theta_{0}\right)^{4} \alpha\right]+\frac{1}{r^{4} \sin ^{4} \theta \sin ^{2} \phi}\left[c_{3}\left(\partial_{4} \Theta_{0}\right)\left(\partial_{3} \Theta_{0}\right)\right.} \\
& \left.+c_{4}\left(\partial_{4} \Theta_{0}\right)^{3}\left(\partial_{3} \Theta_{0}\right) \alpha-c_{4}\left(\partial_{3} \Theta_{0}\right)^{2}-c_{4}\left(\partial_{3} \Theta_{0}\right)^{4} \alpha\right]+\frac{m^{2} c_{4}}{r^{2} \sin ^{2} \theta \sin ^{2} \phi}-\frac{e A_{0}}{z r^{2} \sin ^{2} \theta \sin ^{2} \phi}\left[c_{0}\left(\partial_{4} \Theta_{0}\right)+c_{0}\left(\partial_{4} \Theta_{0}\right)^{3} \alpha\right. \\
& \left.-c_{4}\left(\partial_{0} \Theta_{0}\right)-c_{4}\left(\partial_{0} \Theta_{0}\right)^{3} \alpha-c_{4} e A_{0}-c_{4}\left(\partial_{0} \Theta_{0}\right)^{2} e A_{0} \alpha\right]=0 .
\end{aligned}
$$

[1] S. W. Hawking, Commun. Math. Phys. 43, 199(1975).

[2] M. Sharif, W. Javed, Eur. Phys. J. C 72, 1997(2012).

[3] T. Damoar, R. R uffini, Phys. Rev. D 14, 332(1976).

[4] W. Javed, G. Abbas, R. Ali, Eur. Phys. J. C 77, 296(2017).

[5] A. Övgun, W. Javed, R. Ali, Advance in High Energy Physics. 2018, 11(2018).

[6] W. Javed, R. Ali, G. Abbas, Can. J. Phys. 97, 176(2018).

[7] W. Javed, R. Ali, R. Babar, A. Övgun, Eur. Phys. J. Plus 134, 511(2019).

[8] W. Javed, R. Ali, R. Babar, A. Övgun, Chinese Physics C 144, 015104 (2020).

[9] G. Johnson, JHEP 03, 038(2020).

[10] R. Ali, K. Bamba, S. A. A. Shah, Symmetry. 631, 11(2019).

[11] R. Ali, K. Bamba, M. Asgher, M. F. Malik and S. A. A. Shah, Symmetry. 1165, 12(2020).

[12] R. Ali, M. Asgher, M. F. Malik, Mod. Phys. Lett. A 35, 2050225(2020).

[13] M. Rizwan, M.Z. Ali, A. Övgun, Mod. Phys. Lett. A 34, 1950184(2019).

[14] R. Ali, et al. Int. J. Mod. Phys. D 30, 2150002(2021).

[15] W. Javed, R. Babar, Proceedings of the 15th Marcel Grossmann Meeting, http://robot.icranet.org:8080/store/1380.pdf; ibid. Punjab University Journal of Mathematics 52, 6(2020).

[16] W. Javed, R. Babar, A. Övgün, Mod. Phys. Lett. A 34, 1950057(2019).

[17] W. Javed, R. Babar, Adv. High Energy Phys. 2019, 2759641(2019); ibid. Chinese Journal of Phys. 61, 138(2019).

[18] R. Babar, W. Javed, A. Övgün, Mod. Phys. Lett. A 35, 2050104(2020).

[19] K. Konishi, G. Paffuti, P. Provero, Phys. Lett. B 234, 276(1990).

[20] L. J. Garay, Int. J. Mod. Phys. A 10, 145(1995).

[21] R. Ali, R. Babar, M. Asgher, S. A. A. Shah, Annals of Physics, 432, 168572(2021).

[22] A. Kempf, G. Mangano, R. B. Mann, Phys. Rev. D 52, 1108(1995).

[23] S. Das, E. C. Vagenas, Phys. Rev. Lett. 101, 221301(2008).

[24] Chen, D., Wu, H., Yang, H. Yang, S.: Int. J. Mod. Phys. A 29(2014)1430054.

[25] A. Kempf, J. Phys. A 30, 2093(1997).

[26] F. Brau, J. Phys. A 32, 7691(1999).

[27] A. F. Ali, S. Das, E. C. Vagenas, Phys. Lett. B 678, 497(2009).

[28] A. Övgün, K. Jusufi, Eur. Phys. J. Plus 132, 298(2017).

[29] B. Zhang, Q. Cai and M. S. Zhan, Phys. Lett. B 665, 260(2008).

[30] S. H. Mehdipour, Int. J. Mod. Phys. A 25, 5543(2010).

[31] M. Sharif, G. Abbas, Mod. Phys. Lett. A 26, 23(2011).

[32] L. H. Ling, Sci China-Phys Mech Astron. 55, 11(2012).

[33] C. Z. Liu, Int. J. Theor. Phys. 53, 60(2014). 
[34] G. M. Deng, J. Phys . Conf. Ser. 942, 012008(2017).

[35] G. Gecim, Y. Sucu, Mod. Phys. Lett. B 28, 185164(2018).

[36] A. Addazi, Homa Shababi, Int. J. Mod. Phys. A 35, 2042002(2020).

[37] M. Yi, X. Wu, Phys. Scr. 95, 085008(2020).

[38] A. Addazi, Homa Shababi, Int. J. Geom. Meth. Mod. Phys. 17, 2050130(2020).

[39] B. Khosropour, M. Eghbali, S. Ghorbanali, Gen. Relativ. Gravit. 50, 25(2018).

[40] A. Awad, Gamal Nashed, JCAP. 02, 046(2017).

[41] G. G. L. Nashed, EPL. 105, 10001(2014).

[42] E. Elizalde, G. G. L. Nashed, S. Nojiri, S. D. Odintsov, Eur. Phys. J. C 80, 109(2020).

[43] W. E Hanafy, G. G. L. Nashed, Astrophys. Space Sci. 361, 68(2016).

[44] T. Shirafuji, G. G. L. Nashed, Prog. Theor. Phys. 98, 1355(1997)

[45] M. Sharif, W. Javed, Proceedings of the 3rd Galileoâ€ $€^{\prime \prime} \mathrm{Xu}$ Guangqi Meeting, Int. J. Mod. Phys.: Conference Series, 23, 271(2013).

[46] S. Bose, N. Dadhich, Phys. Rev. D 60, 064010(1999).

[47] X. Q. Li, G. R Chen, Phys. Lett. B 751, 34(2015).

[48] X. Q. Li, Phys. Lett. B 763, 80(2016).

[49] X. Q. Li, Phys. Lett. B 763, 80(2016).

[50] M. Dehghani Â. A. Farmany, Int. J. Theor. Phys. 49, 1633(2010).

[51] P. Wang, H. Yang, S. Ying, Int. J. Theor. Phys. 55, 2633(2016).

[52] R. Banerjee, B. R. Majhi, Journal of High Energy Phys. 2008, 095(2008). 